\title{
Meningeal carcinomatosis: a retrospective analysis of seventy-seven cases
}

\author{
Feng-Na Chu, Yue Lang, Xiao-Min Sun, Li Cui \\ Neuroscience Center, the First Hospital of Jilin University, Jilin University, Changchun 130021, Jilin, China.
}

Correspondence to: Prof. Li Cui, Neuroscience Center, the First Hospital of Jilin University, Jilin University, Changchun 130021, Jilin, China. E-mail: chuili1967@126.com

How to cite this article: Chu FN, Lang Y, Sun XM, Cui L. Meningeal carcinomatosis: a retrospective analysis of seventy-seven cases. Neuroimmunol Neuroinflammation 2017;4:1-5.

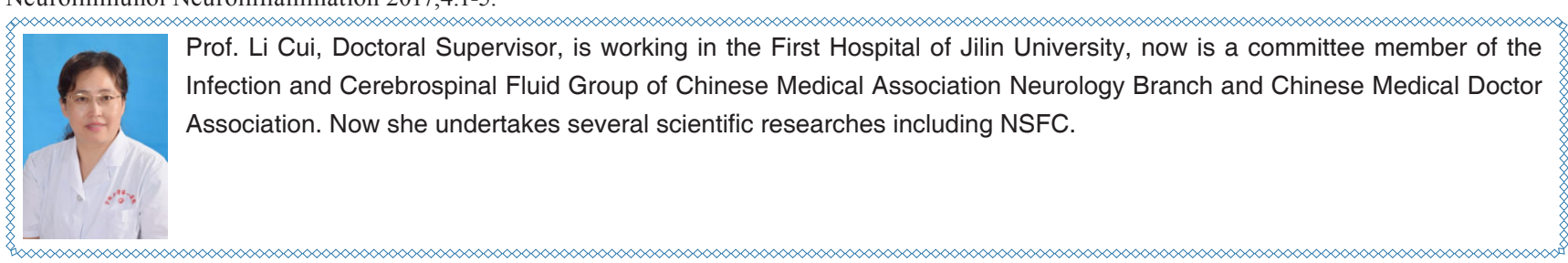

\section{Article history:}

Received: 20-05-2016

Accepted: 19-12-2016

Published: 20-01-2017

\section{Key words:}

Meningeal carcinomatosis, cerebrospinal fluid, magnetic resonance imaging, therapy

\section{ABSTRAC T}

\begin{abstract}
Aim: Meningeal carcinomatosis is a special type of malignant tumor characterized by short survival and poor prognosis. In the present study, the authors aim to analyze the clinical, laboratory data and prognosis of meningeal carcinomatosis patients. Methods: The authors enrolled 77 cases of meningeal carcinomatosis from 2003 to 2013 in the First Hospital of Jilin University. The clinical data including age, gender, symptoms at onset, clinical manifestations, primary tumors and the laboratory data including cerebrospinal fluid (CSF), tumor markers as well as the imaging data were analyzed. The interval between the onset of primary tumor and the onset of central nervous symptoms, treatments and survival time were also analyzed. Results: The onset of meningeal carcinomatosis was usually acute $(46.2 \%)$ or subacute $(39.0 \%)$. The most frequent symptom at onset was intracranial hypertension (70.1\%). Symptoms such as headache, vomit and high lumbar puncture intracranial pressure was observed in $56 \%$ of cases during the course of the disease. CSF abnormalities such as higher protein concentration (73.4\%), more CSF pleocitosis (57.1\%) and lower glucose levels (48.4\%) were found in 95.3\% of meningeal carcinomatosis patients. Non-contrast enhanced cerebral magnetic resonance imaging (MRI) showed that $13.2 \%$ patients had abnormal meningeal changes while in the enhancement scan $35.3 \%$ patients showed changes. The serum tumor markers increased in $84 \%$ of the patients. There were no differences regarding the mean survival between patients who received intrathecal chemotherapy and those who received brain radiotherapy or supportive treatment. Conclusion: The most common clinical manifestation of meningeal carcinomatosis is intracranial hypertension. The most common primary tumor is lung cancer, followed by gastric cancer and breast cancer. The linear enhancement of meningeal on the MRI scan is of great importance for the diagnosis of meningeal carcinomatosis.
\end{abstract} (i) (2) This is an open access article distributed under the terms of the Creative Commons Attributioncommercially, as long as the author is credited and the new creations are licensed under the identical terms. 


\section{INTRODUCTION}

Meningeal carcinomatosis (MC) is caused by the spread of cancer cells to the leptomeninges and by their dissemination within the cerebrospinal fluid (CSF). ${ }^{[1]} \mathrm{MC}$ prognosis is usually poor with a short survival. ${ }^{[2]}$ In recent years, several studies focused on the early diagnosis of MC. CSF cytology combined with CSF tumor markers were reported to have great value for early diagnosis and for addressing the origin of meningeal carcinomatos. ${ }^{[3]}$

\section{METHODS}

\section{Patient information}

From 2003 to 2013, patients who admitted to Department of Neurology at the First Hospital of Jilin University and fulfilled the diagnostic criteria of $\mathrm{MC}$ were enrolled. ${ }^{[4]}$ This retrospective study was approved by the ethics committee of the First Hospital of Jilin University, Changchun, China.

\section{Clinical data}

A total of 77 patients were enrolled in our study. We collected data about the gender, age of onset, type of onset, initial symptoms, clinical manifestations, physical examination, time and frequency of lumbar puncture, CSF pressure, routine biochemical and cytological examination results, CSF tumor markers, imaging examinations [head computed tomography, magnetic resonance imaging (MRI) scan and enhanced scan], serum tumor markers, primary tumors, the interval between primary tumor and central nervous symptoms, treatment methods and the survival time.

The above data was provided by the First Hospital of Jilin University Clinical Laboratory and Imaging Department.

\section{Statistics}

Statistical analysis was performed using SPSS version
17.0 software (SPSS, IBM, West Grove, PA, USA). Categorical data were presented as proportions, while continuous data were presented as means and standard deviations of means or interquartile ranges depending on the distribution of the data. Differences in proportions were tested by the Chi-square tests and differences in continuous variables were tested by student $t$-tests. For all statistical tests, $P<0.05$ was considered to be significant.

\section{RESULTS}

\section{The demographic data of MC patients}

Among the 77 patients enrolled to the study, 35 were males and 42 were females. The median age at diagnosis was 55 years old (ranging from 2 to 76 years old).

\section{Clinical manifestations and physical examination findings}

In this patient cohort, there were $36(46.2 \%)$ cases with acute onset, $30(39 \%)$ cases with subacute onset and $11(14.3 \%)$ cases with chronic onset. Most patients were admitted to hospital with increased intracranial pressure symptoms such as headache, nausea and vomit. Some patients showed cranial nerve or brain parenchyma damage symptoms and severe cases presented with unconsciousness or cerebralhernia. Physical examination revealed meningeal irritation, pyramidal signs, cranial nerve paralysis, etc. The main symptoms and signs are shown in Table 1.

\section{Lumbar puncture and CSF routine test}

Intracranial pressure, CSF cytology, tumor markers and immunoglobulin

The high lumbar puncture intracranial pressure was observed in $56 \%(32 / 57)$ of cases, and $21.1 \%$ of them showed over $400 \mathrm{mmH}_{2} \mathrm{O}$. The distribution of the intracranial pressure is shown in Figure 1.

In the first CSF cytology examination, 82.2\% (60/73)

Table 1: Symptoms and signs of meningeal carcinomatosis patients

\begin{tabular}{|c|c|c|c|c|c|}
\hline $\begin{array}{l}\text { CNS involvement } \\
\text { symptoms and signs }\end{array}$ & $\begin{array}{l}\text { Number of } \\
\text { cases (\%) }\end{array}$ & $\begin{array}{l}\text { Cranial nerve } \\
\text { involvement }\end{array}$ & $\begin{array}{l}\text { Number of } \\
\text { cases (\%) }\end{array}$ & $\begin{array}{l}\text { Spinal and PNS } \\
\text { involvement }\end{array}$ & $\begin{array}{l}\text { Number of } \\
\text { cases (\%) }\end{array}$ \\
\hline $\begin{array}{l}\text { Headache, nausea and } \\
\text { vomiting }\end{array}$ & $54(70.1)$ & Abducens nerve palsy & $10(13.0)$ & $\begin{array}{l}\text { Bilateral limbs weakness } \\
\text { or paresthesia }\end{array}$ & $12(15.6)$ \\
\hline Dizziness & $21(27.3)$ & Oculomotor nerve paralysis & $10(13.0)$ & $\begin{array}{l}\text { Tendon reflexes diminish } \\
\text { or disappear }\end{array}$ & $11(14.3)$ \\
\hline Hyperspasmia & $11(14.3)$ & Facial paralysis & $10(13.0)$ & Neck and shoulder pain & $5(6.5)$ \\
\hline Mental disorders & $5(6.5)$ & Double vision & $7(9.1)$ & Unsteady gait & $3(3.9)$ \\
\hline $\begin{array}{l}\text { Disturbance of } \\
\text { consciousness }\end{array}$ & $3(3.9)$ & Hypoglossal nerve palsy & $7(9.1)$ & $\begin{array}{l}\text { Rectal bladder } \\
\text { dysfunction }\end{array}$ & $1(1.3)$ \\
\hline Papilledema & $4(5.2)$ & Decreased vision & $4(5.2)$ & Saddle area sensory loss & $1(1.3)$ \\
\hline Pyramidal signs & $20(26.0)$ & Hearing loss & $4(5.2)$ & Lasegue positive sign & $1(1.3)$ \\
\hline Meningeal irritation & $41(53.2)$ & Pronunciation or dysphagia & $4(5.2)$ & & \\
\hline
\end{tabular}

CNS: central nervous system; PNS: peripheral nervous system 
cases showed abnormal cells; while in $8.2 \%(6 / 73)$ cases abnormal cells were found through a second time lumbar puncture; only in one case tumor cells were found in the third lumbar puncture. Cell morphology analysis revelaed that 14 cases showed adenocarcinoma cells. The remaining cases were characterized by an increase in cell size, irregular shape, cell body stain and darker cytoplasm. Cell membranes were incomplete and with protrusions. Nuclear cytoplasm ratio increased, and nucleus were centered or showed deviation, occasionally they were double-nucleated. In some cells, cytoplasmic vacuoles were observed near the membrane. Some mitotic cells could also be observed. The cytology results for different cancers are shown in Figure 2. Only 3 cases of the CSF tumor markers were checked, and all the results were abnormal. Patients with elevated IgG accounted for $72.7 \%$ (16/22).

CSF routine biochemical examination was found abnormal in $95.3 \%(61 / 64)$ patients. High protein accounted for $74 \%$ (46/62) (normal range $0.15-0.45 \mathrm{~g} / \mathrm{L}$ ), reduced glucose was $45 \%$ (27/60) (normal range 2.3$4.1 \mathrm{mmol} / \mathrm{L})$, reduced chlorine accounted for $38 \%$ (23/60) (normal range 119-129 mmol/L), elevated white blood cell count was $59.7 \%$ (37/62) (normal range 0-8 $\times 10^{6} / \mathrm{L}$ ), which was given priority to mononuclear cells.

\section{Tumor marker changes in MC patients}

The rate of abnormal serum tumor markers was $84 \%$. Sixty-eight percent $(17 / 25)$ of the patients had increased carcinoembryonic antigen. In 44\% (11/25) of the patients CYFRA21-1 increased. The rate of

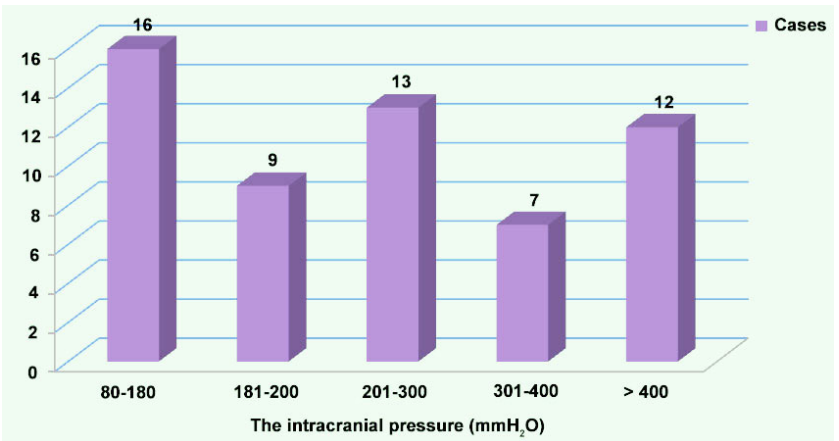

Figure 1: The distribution of the intracranial pressure

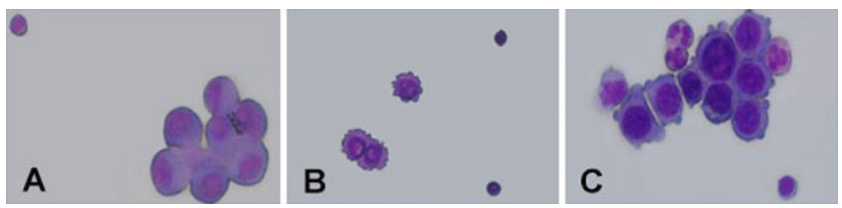

Figure 2: Cytology results for three types of cancer patient. (A) One case of lung cancer meningeal carcinomatosis cerebrospinal fluid cytology test result; $(B)$ one case of breast cancer meningeal carcinomatosis cerebrospinal fluid cytology test result; $(C)$ one case of gastric meningeal carcinomatosis cerebrospinal fluid cytology test result the increased CA125 was $32 \%(8 / 25) .32 \%(8 / 25)$ of the patients had increased CA199. In $28 \%(7 / 25)$ of the patients NSE increase was observed. The rate of the increased CA153 was $24 \%(6 / 25)$. There were $16 \%(4 / 25)$ of the patients who had increased CA724. Four percent $(1 / 25)$ of the patients' alpha-fetoprotein increased. The rate of elevated blood sedimentation was $66.7 \%(10 / 15)$.

\section{Imaging findings in MC patients}

Twenty-four cases had head computed tomography examinations. Among these, one of them showed that meninges thickened significantly. One case showed the expansion of the ventricles and hydrocephalus, the others had no significant abnormalities. Fiftythree cases had head MRI scan examinations and 17 (32.1\%) of them were abnormal. A total of $12(35.3 \%)$ cases had meningeal reinforcement in the 34 cases of enhanced scan.

\section{Primary tumors}

The most frequent primary tumor in our study was lung cancer $(35 / 77,58.3 \%)$, followed by gastric cancer $(10 / 77,16.7 \%)$, breast cancer $(6 / 77,10 \%)$, melanoma $(3 / 77,5 \%)$ and non-hodgkin's lymphoma (2/77, 3.3\%). In addition, one patient presented with primary lesion in ovarian and one presented with colon cancer. In one case the primary tumor was nasopharyngeal carcinoma, and in another it was acute lymphocytic leukemia. Seventeen (22.1\%) had no primary tumor. The interval from diagnosis of primary tumor to the onset of central nervous system (CNS) symptoms was also analyzed: 45 cases $(58.4 \%)$ initially presented with CNS symptoms without history of tumor; $26 \%$ (20/77) patients developed CNS symptoms when the primary tumor had been diagnosed for no more than one year; $6.5 \%(5 / 77)$ patients experienced CNS symptoms at one to two years after the diagnosis of primary tumor; $9.1 \%$ (7/77) patients did not experience the CNS symptoms until the primary tumor has been diagnosed for more than two years.

\section{Treatment and survival}

Thirteen patients received intrathecal chemotherapy (with methotrexate or cytarabine or dexamethasone) and radiotherapy treatment and other 13 patients received only symptomatic and supportive treatment. The remaining patients were lost during the follow-up. The mean survival period in the radiotherapy and chemotherapy treatment group was $24.77 \pm 22.80$ weeks, whereas in the symptomatic and supportive treatment group it was $12.46 \pm 18.00$ weeks $(P=0.14)$. There was no statistically significant difference between the survivals of these two groups. 


\section{DISCUSSION}

MC is a special type of metastatic carcinoma of the central nervous system with a incidence of $5-10 \%$ in all solid tumors. ${ }^{[5,6]}$ As a gradual increase of incidence and a high mortality have been observed over time, early diagnosis is crucial. Due to its diverse clinical manifestations, it is difficult to make a diagnosis only with imaging examinations and CSF cytology is necessary to find abnormal cells.

At onset, the age is similar between $\mathrm{MC}$ and other tumors. In fact, MC can be found at any age, but it is more common in the elderly. Most patients present with acute or subacute onset, while a small number of patients with primary tumors may be in chronic onset. Since cancer cells can move into the CSF and infiltrate the dura and the brain parenchyma, clinical evidence of the disease could appear as symptoms of meninges and brain parenchyma damage,$^{[7]}$ cranial nerve palsy and spinal nerve root symptoms. ${ }^{[8]}$ The most frequent initial symptom is intracranial hypertension such as headache, nausea and vomit. The main reason for headache is the increasing intracranial pressure caused by cancerous obstruction of CSF circulation pathway. Besides, the meninges irritation is partly responsible for headache.

In this study, we found $11(14.3 \%)$ cases of convulsions, $5(6.5 \%)$ of mental disorders such as illusion and personality change, $3(3.9 \%)$ of conscious disorder and other CNS damaged symptoms. The common signs include $41(53.2 \%)$ cases of meningeal stimulation and $20(26 \%)$ cases of positive pyramidal tract, etc. The most common damaged nerves include abducens nerve, oculomotor nerve and facial nerve, and each type of damaged nerve accounts for 10 cases $(13 \%)$ in our study. The other cranial nerves such as optic nerve, auditory nerve, hypoglossal nerve, glossopharyngeal nerve also can be involved, which was similar with what already described in the literature. ${ }^{[9]}$ In this study, there were $12(15.6 \%)$ patients with bilateral limbs weakness or paresthesia, 11 (14.3\%) patients with tendon reflexes diminished or disappeared, 5 (6.5\%) patients with the neck shoulder pain which is the effect of the infiltration of tumor cells and the irritation of the spinal nerve root. In addition, there was one case with rectal bladder dysfunction and saddle area sensory deficiency. The Kernig sign and the Lasegue sign may be positive in the physical examination.

The cerebral vascular endothelial permeability increases when the meninges are stimulated by tumor cells and produce various chemicals, at the same time the CSF circulation path may be blocked by tumor cells. All of above can cause increase in the intracranial pressure. This is confirmed by lumbar puncture measurement. Regular and biochemical tests were used in 62 cases. The protein level and white blood cell count in CSF were found elevated in $47(47 / 62,73.4 \%)$ cases and $36(36 / 62,57.1 \%)$ cases, respectively. The obstruction of CSF circulation path, the disruption of the bloodbrain barrier and the increase of vascular endothelial permeability may cause the protein extravasation and diapedesis. Interestingly, 30 (30/62, 48.4\%) cases showed a lower CSF glucose content than normal, which could be the result of glucose consumption caused by the proliferation and strong metabolism of tumor cells. In addition, as it is difficult for blood glucose to pass through the blood brain barrier, the CSF glucose lacks the timely supply from blood, which is another reason for the drop in glucose content. ${ }^{[10]}$ In $26(41.9 \%)$ cases lower CSF chloride value was observed. A lot of glycolysis produced a great amount of acid metabolites, facilitating the CSF chloride decline (as the chloride reduces easily in acid conditions). At the same time, loss of large amount of gastric acid after frequent vomiting is another reason for chloride reduction. So the overall abnormal rate of protein, glucose, chloride and white cells in CSF was high.

Seventy-three cases underwent CSF cytology examination. Tumor cells in the CSF were found by one lumbar puncture and two lumbar punctures in 60 cases $(82.2 \%)$ and 6 cases $(8.2 \%)$, respectively. Only in one case tumor cells were not found until the third time lumbar puncture. Enough samples combined with accurate assessment from clinical physicians would improve the diagnostic rate. ${ }^{[11]}$ Only in 3 cases, CSF tumor markers were abnormal. It is difficult to diagnose the source of tumor cells on the basis of cytology morphology. We can combine cytology examination with CSF tumor markers to diagnose the properties and origin of tumor cells. ${ }^{[12]}$

It is difficult to diagnose MC through standard MRI scan. But we can find some indirect signs such as ventricular expansion, hydrocephalus, etc. There are a lot of dural blood capillaries in normal humans, but there is no blood brain barrier because lack of close connection between endothelial cells. Although the capillaries of leptomeninges are fewer than dura, the blood brain barrier exists because there are lots of close connection between endothelial cells. ${ }^{[13]}$ The normal dura may be strengthened during head MRI enhanced scanning, but it does not show nodular or hybrid reinforcement. MC also destroys blood brain barrier, in fact the majority of patients shows dural abnormal reinforcement such as nodositas, line type and mixed type. A small number of patients have no 
obvious reinforcement. The leptomeningeal abnormal reinforcement may be due to the direct infiltration of tumor cells from dura or from choroid plexus. Enhanced scan can discover more abnormal meninges, which play an important role in the auxiliary diagnosis of MC.

Serum tumor markers have important significance for the source of the tumor. Tumor markers can have different levels in different clinical periods and can reflect the dynamic changes, recurrence, metastasis, or treatment effect of the tumor. The abnormal rate of serum tumor markers is $84 \%$ in our study. The erythrocyte sedimentation rate, in some physiological conditions, such as anemia, menstrual period, after three months of pregnancy, infection, malignant tumor, tissue necrosis and other diseases, was found increased. The change is not specific, but has great value for malignant tumors fast development.

Most patients with MC have primary tumor. However, in some patients with CNS damage as the first symptom no primary tumor is found, or is found only after a period of time. In this study, primary tumor incidence in patients is consistent with the incidence of $\mathrm{MC}^{[4]}$ in China and the most common is lung cancer.

Literature reported that untreated patients with $M C$ survive for 4-6 weeks whereas patients undergoing regular treatment survive for $4-6$ months. ${ }^{[14]}$ In this study, the difference was not statistically significant when the survival of patients with radiotherapy and chemotherapy was compared with symptomatic treatment patients. Although patients with $\mathrm{MC}$ received intrathecal injection of chemotherapy and whole brain radiotherapy, the treatment did not improve survival. Because MC is considered the terminal stage of the disease, concurrent chemoradiation has no impact on patient outcome, but can improve clinical symptoms. ${ }^{[15]}$

In this study, the clinical manifestations of 77 patients are diverse, but most of them are characterized by increased intracranial pressure symptoms such as headache, nausea and vomit. Therefore, when patients have above symptoms, we should be vigilant about MC and improve the head MRI and enhanced MRI scan, if necessary. CSF cytological examination is the gold standard of the diagnosis of MC. Because CSF tumor marker has not been regarded as a standard criterion of diagnosis and due to the lack of a recognized standard reference value, its application is still restricted. However, since tumor markers have significant implications for primary tumor source, combining this with serum tumor markers examination would contribute to identify the source of tumor cells. Through the analysis of MC, we hope to improve the early diagnostic rate of the disease and help clinicians develop a detailed and suitable treatment plan for patients to increase benefit.

\section{Financial support and sponsorship Nil.}

\section{Conflicts of interest}

There are no conflicts of interest.

\section{Patient consent}

Informed consent was obtained from all individual participants included in the study.

\section{Ethics approval}

This study was approved by the ethics committee of the First Hospital of Jilin University.

\section{REFERENCES}

1. Mittica G, Senetta R, Richiardi L, Rudà R, Coda R, Castellano I, Sapino A, Cassoni P. Meningeal carcinomatosis underdiagnosis and overestimation: incidence in a large consecutive and unselected population of breast cancer patients. BMC Cancer 2015;15:1021.

2. Thiel E, Gleissner B. Neoplastic meningitis. Dtsch Arztebl 2006;103:2559-65.

3. Chamberlain MC. Neoplastic meningitis. Oncologist 2008;13:967-77.

4. DeAngelis LM. Diagnosis and treatment of leptomeningeal metastasis. Educational Book Am Soc Clini Oncol 2002;369:374.

5. Van Horn A, Chamberlain MC. Neoplastic meningitis. J Support Oncol 2012;10:45-53.

6. Chamberlain MC. Leptomeningeal metastasis. Curr Opin Oncol 2010;22:627-35

7. Olson ME, Chernik NL, Posner JB. Infiltration of the leptomeninges by systemic cancer. A clinical and pathologic study. Arch Neurol 1974;30:122-37.

8. Kato Y, Takeda H, Dembo T, Fukuoka T, Tanahashi N. Progressive multiple cranial nerve palsies as the presenting symptom of meningeal carcinomatosis from occult colon adenocarcinoma. Intern Med 2012;51:795-7.

9. Nardone R, Herz M, Egarter-Vigl E, Tezzon F. Isolated oculomotor nerve palsy as the presenting clinical manifestation of a meningeal carcinomatosis: a case report. Neurol Sci 2006;27:288-90.

10. Taillibert S, Laigle-Donadey F, Chodkiewicz C, Sanson M, HoangXuan K, Delattre JY. Leptomeningeal metastases from solid malignancy: a review. J Neurooncol 2005;75:85-99.

11. Gao W, Li Z, Wang LM, Lian F, Liu CC, Lu DH, Piao YS. The clinical and pathological features of 85 cases with positive cerebrospinal fluid cytology by thin-layer centrifugal cytological test. Zhonghua Nei Ke Za Zhi 2016;55:945-9. (in Chinese)

12. Shi Q, Pu CQ, Huang XS, Tian CL, Cao XT. Optimal cut-off values for tumor markers in cerebrospinal fluid with ROC curve analysis. Front Biosci (Elite Ed) 2011;3:1259-64.

13. Lyu Y, Li C, Zhou X, He W, Lyu P, Zhou Z, Wang D, Ning F, Wang Y. MR image analysis of 147 cases of meningeal tuberculosis. Zhonghua Jie He He Hu Xi Za Zhi 2015;38:815-20. (in Chinese)

14. Lin N, Dunn IF, Glantz M, Allison DL, Jensen R, Johnson MD, Friedlander RM, KesariS. Benefit of ventriculoperitoneal cerebrospinal fluid shunting and intrathecal chemotherapy in neoplastic meningitis: a retrospective, case-controlled study. J Neurosurg 2011;115:730-6.

15. Palma JA, Fernandez-Torron R, Esteve-Belloch P, Fontes-Villalba A, Hernandez A, Fernandez-Hidalgo O, Gallego Perez-Larraya J, Martinez-Vila E. Leptomeningeal carcinomatosis: prognostic value of clinical, cerebrospinal fluid, and neuroimaging features. Clin Neurol Neurosurg 2013;115:19-25. 